\title{
THE INFLUENCE OF PHYSIOTHERAPY PROGRAM ON ACTIVITIES IN DAILY LIVING IN PATIENTS WITH DISC HERNIATION AFTER SYRGERY
}

\author{
Tsvetelina Bizheva \\ National Sports Academy “Vassil Levski”, Sofia, Bulgaria
}

\begin{abstract}
The purpose of this study is to evaluate the effect of one-month specialized physiotherapy program on activities of daily live in patients with disc herniation after spinal surgery. Twenty patients with disc herniation after surgery were studied. They were divided into two groups, a control group (CG $n=10)$ and an experimental group (EG $n=10)$. The assessment was made on the day of discharge and one month after surgery. The Activities in Daily Living (ADL) were examined with Oswestry disability index and Functional gait assessment test. Gait training and exercises imitating the ADL at home for one month after surgery were given to the EG. The CG was received standard physical therapy program. The results show that the highest improvement in the ADL was seen in sitting, standing and social life. The biggest difference in walking was observed in walking in different directions and walking backward. Discussion: Postoperative management of patients undergoing spinal surgeries emphasize suitable positioning coupled with patient education and advice on reinforcing self-management and on building activities appropriately. Proper implementation of the ADL lead to faster recovery and increase the independence in daily living. Conclusions: The performance of ADL exercises combined with gait training may significantly improve the possibilities for walking and increace the independence in daily living in patients after lumbar disc herniation surgery.
\end{abstract}

Key words: physiotherapy, disc herniation, surgery.

\section{INTRODUCTION}

Low back pain is becoming an epidemiological problem and one of the most common reasons for visits to a doctor's surgery, generating enormous social costs. It is estimated that about two-thirds of adults in the USA may be affected by some form of low back pain in their lifetime. Symptomatic lumbar disc herniation is a common spinal disease affecting $1 \%-3 \%$ of general population, but only $15 \%-20 \%$ of these cases require operative intervention (Kashani et al., 2015). The incidences of degenerative diseases of the lumbar spine and spinal surgery are both increasing as the population becomes more elderly (Mokhtar, McCombe, 2010). In Poland, sciatica ranks in 4th place in the list of the main causes of work absenteeism (Gawda et al., 2015). Most patients with lumbar disc herniation are often accompanied by pain in one or both lower extremities, limited physical activity, decreased ability to live and reduced social activities. The main clinical symptoms of this disease are low back pain, radiation pain of lower limbs, numbness, which seriously affects the normal life and work of patients. The disease has a high incidence, a long course, easy recurrence and difficult to cure (Xiaoping et al., 2019) Patients had been exhibiting symptoms for many years, reducing ADL (Kazufumi et al., 2017).

Rates of spinal surgery differ across countries and within one country. About 12,000 operations for herniated lumbar discs are performed in the Netherlands each year. Rates in the United States are $30 \%$ higher than in the Netherlands, $50-60 \%$ higher than in Canada and $80 \%$ higher than in the UK (Oosterhuis et al., 2013).

Studies have reported that after surgery, patients are prone to severe pain, which affects their ability to perform ADLs (Xiaoli et al., 2019). The surgical removal of the disc does not resolve the whole pathological process of the lumbar disorder, and this is the reason for the importance of post-operative exercise treatment programs for rehabilitation (Ju et al., 2012).

The aim of this study is to evaluate the effect of onemonth specialized physiotherapy program on activities of daily live and gait in patients with disc herniation after spinal surgery.

\section{METHODS}

Twenty patients with disc herniation after surgery 
voluntarily attended and treated in the Department of neurosurgery of the University Hospital Sofiamed - Sofia were studied. Patients were randomly divided into two groups, a control group (CG $\mathrm{n}=10)$ and an experimental group (EG $\mathrm{n}=10)$. All patients had similar impairments and functional limitations. The assessment was made on the day of discharge and one month after surgery. The Activities in Daily Living (ADL) were examined with Oswestry disability index (ODI). The gait was examined with a Functional gait assessment (FGA) test. From the first day after discharge all patients performed physical therapy three times in week, for 30 minutes. The patients from the experimental group performed a program including exercises to improve the adaptability of walking and ADL activities for one month. Gait training exercises include: walking with a change in speed, walking with both horizontal and vertical head movement, crossing over an object, walking backwards and walking with closed eyes. The patient also performed activi- ties that are as close as possible to those in everyday life: getting up from bed, putting on shoes, sitting in a chair, getting up from a chair, lifting, getting objects above the head, washing dishes, driving a car, getting a shower and others. The CG was received standard physical therapy program with exercises and instructions how to continue exercises at home and to resume daily activities gradually.

\section{RESULTS}

There were improvements in all functional aspects of the gait in the first month after treatment for both groups (Figure 1). The significant difference between the groups was seen in indicators: gait end pivot turn with 0.7 points and walking backwards with 0.6 points (Figure 2). The rest of the indicators improve with 0,2 to 0.4 points. The overall point for FGA at the beginning were 14 points for the CG and 15 points for EG. After one month there was an improvement in the EG to 21.65 points, while the CG had 20.5 points (Figure 3).

\section{FGA - subscales}

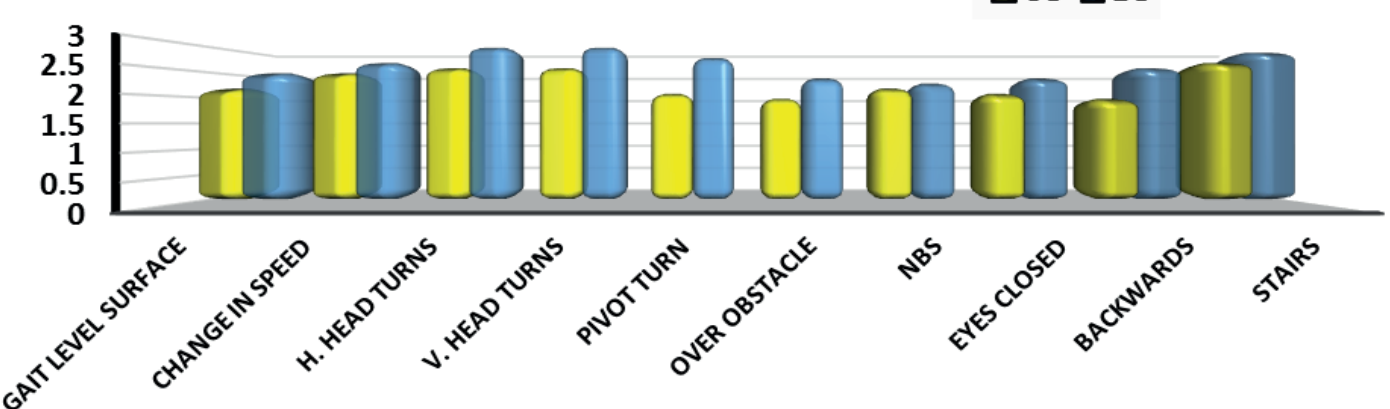

\begin{tabular}{|l|c|c|c|c|c|c|c|c|c|c|}
\cline { 2 - 10 } \multicolumn{1}{c|}{} & $\begin{array}{c}\text { gait level } \\
\text { surface }\end{array}$ & $\begin{array}{c}\text { Change in } \\
\text { speed }\end{array}$ & $\begin{array}{c}\text { H. head } \\
\text { turns }\end{array}$ & $\begin{array}{c}\text { V. head } \\
\text { turns }\end{array}$ & pivot turn & $\begin{array}{c}\text { over } \\
\text { obstacle }\end{array}$ & NBS & $\begin{array}{c}\text { eyes } \\
\text { closed }\end{array}$ & backwards & stairs \\
\hline 口CG & 2.2 & 2.5 & 2.6 & 2.6 & 2.1 & 2 & 2.2 & 2.1 & 2 & 2.7 \\
\hline 口EG & 2.5 & 2.7 & 3 & 3 & 2.8 & 2.4 & 2.3 & 2.4 & 2.6 & 2.9 \\
\hline
\end{tabular}

Figure 1. Changes in the subscales of FGA in CG and EG after one month after treatment

FGA - difference

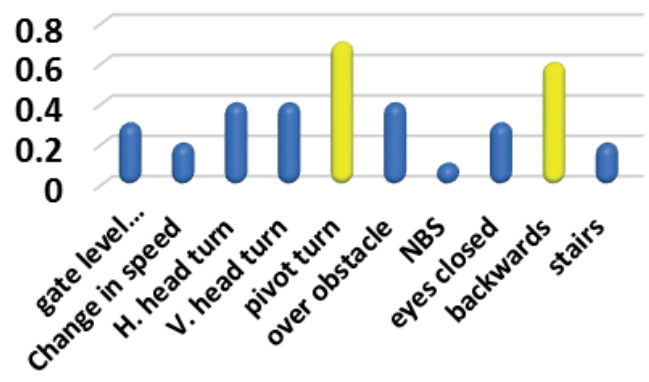

Figure 2. Significant difference in FGA between CG and EG one month after treatment
FGA -total score

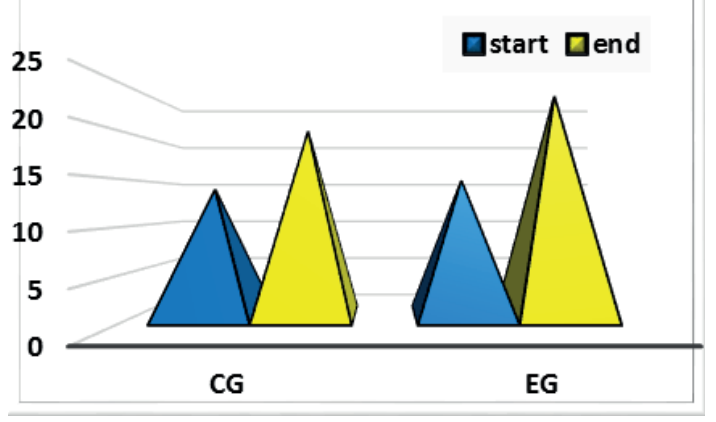

Figure 3. Changes in the FGA overall points in CG and EG before and after applied physical therapy 
In the activities in daily living there were improvements on the first month after treatment for both groups (Figure 4). Significant difference between the groups was seen in personal care, walking, sitting, standing and social life (Figure 5). The overall score for LBP at the beginning were 17.1 points for the CG and 18.1 points for EG. After one month there was a significant improvement in the EG to 6.9 points, while the CG had 11.2 points (Figure 6).

\section{LBP test after treathment}

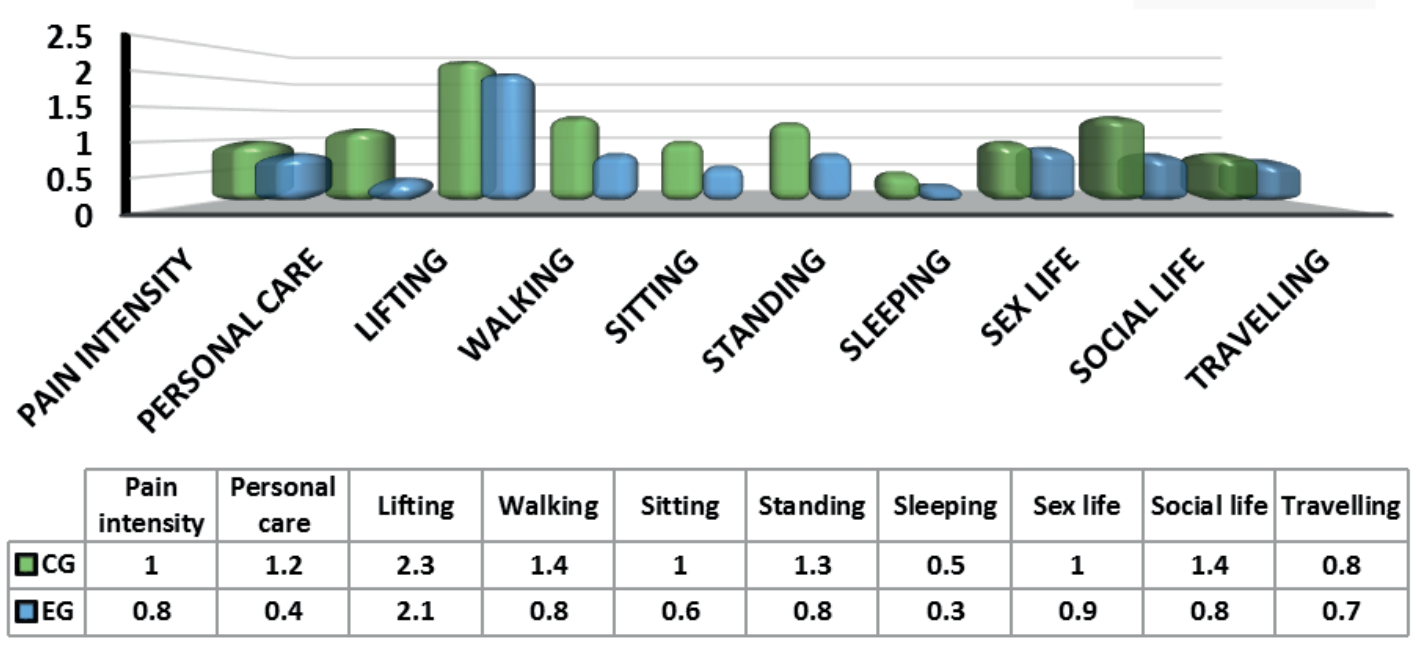

Figure 4. Changes in the subscales of LBP in CG and EG one month after treatment

\section{LBP - difference}

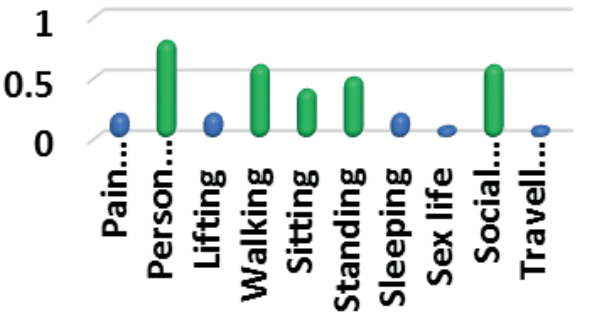

Figure 5. Significant difference in LBP between $C G$ and EG one month after treatment

\section{DISCUSSION}

Care after discharge shows greater variability. Rehabilitation guidelines for postoperative management of patients undergoing spinal surgeries emphasize suitable positioning coupled with patient education and advice on sitting related to patient's function, on reinforcing self-management, and on building activities appropriately. According to some researches (Marchand et al., 2016), rehabilitation programs, for the most part, included some form of strengthening exercises alone or in combination with stabilization exercises, aerobic conditioning, stretching, or education. In our one-month specialized physiotherapy program, we used training in proper motion and performance of ADL imme-

\section{LBP - total score}

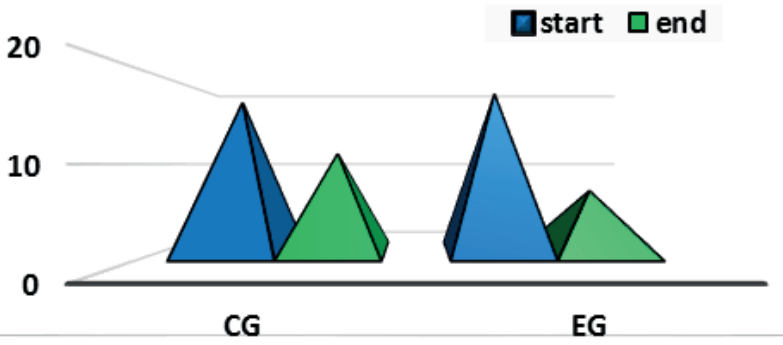

Figure 6. Changes in the LBP overall points in CG and EG before and after applied physical therapy

diately after the discharge. Proper implementation of the ADL and avoiding bending and rotation lead to faster recovery and increase the independence in daily living. Thus patients gained confidence and self-esteem in their movements.

Recent studies show that advice regarding walking and return to physical activity are a key component of physiotherapy intervention immediately following lumbar surgery. Studies on the effects of walking exercises for treating post-surgical lumbar disc herniation are not many. According to Sokunbi et al., 2015, one of the most popular types of exercise equipment used by physiotherapist in rehabilitation is the treadmill, which provides a straight- 
forward, efficient aerobic workout. We believe, that performing exercises to respond on motor reactions while walking, like, walking with head movement, crossing over an object, walking backwards and walking with closed eyes is an easy and affordable way to exercise walking in the first month in patients with disc herniation after surgery.

The results of our study are the same as those of (Ozkara et al., 2015), which considered that postoperative exercise program starting immediately after surgery can improve pain, disability, and spinal function.

\section{CONCLUSIONS}

A wide range of objective and subjective outcomes is used to document changes after active rehabilitation. Program components include both active and assisted interventions combined with various means of education and discussion. Multimodal rehabilitation protocols after lumbar surgery may be used to improve patient-reported and objective outcome measures such as pain, disability, and physical function (Marchand et al., 2016). The results of previous research together with this study's findings suggest that the performance of ADL exercises combined with gait training may significantly improve the possibilities for walking and increase the independence in daily living in patients after lumbar disc herniation surgery. A more in-depth study of the different walking elements, as well as the factors that specifically affect the gait and ADL in patients with disc herniation after surgery need to be done.

\section{REFERENCES}

Gawda, P, Graniczka, M, Pawlak, H., Cybulski, M, Kiełbus, M, Majcher, P, Buczaj, A, Buczaj, M. (2015). Evaluation of influence of stretching therapy and ergonomic factors on postural control in patients with chronic non-specific low back pain. Annals of Agricultural and Environmental Medicine 2015, Vol 22, No 1, 142-146

Ju, S, Park, G, Kim, E. (2012). Effects of an exercise treatment program on lumbar extensor muscle strength and pain of rehabilitation patients recovering from lumbar disc herniation surgery. J Phys Ther Sci. 2012;24:515518.

Kashani, F, Hejrati, H, Ariamanesh, S. (2016). Ten Important Tips in Treating a Patient with Lumbar Disc Herniation. Asian Spine J 2016;10(5):955-963

Kazufumi, M., Eiki, T., Kazuhiro, I. and Shigenobu, S. (2017). Quality of life one year after lumbar spinal fusion. Phys Ther Res 20: 36-43, 2017

Marchand, A., Shaughnessy, J., Châtillon, C., Sorra, K., Descarreaux, M. (2016). Journal of Manipulative and Physiological Therapeutics, Volume 39, Issue 9, November-December 2016, Pages 668-692

Mokhtar, SA., McCombe, P. (2010). Health-related quality of life: a comparison of outcomes after lumbar fusion for degenerative spondylolisthesis with large joint replacement surgery and population norms. Spine J. 2010; 10: $306-312$

Ozkara, G., Ozgen, M., Ozkara, E., Armagan, O., Arslantas, A. and Atasoy, M. (2015). Effectiveness of physical therapy and rehabilitation programs starting immediately after lumbar disc surgery. Turkis Neurosurgery 2015; 25: 372-379

Oosterhuis, T., Tulder, M., Peul, W., Bosmans, J., Lankamp, C., Smakman, L., Arts, M. and Ostelo, R. (2013). Effectiveness and cost-effectiveness of rehabilitation after lumbar disc surgery (REALISE): design of a randomised controlled trial. BMC Musculoskeletal Disorders 2013, 14:124 Available at: http://www.biomedcentral.com/1471-2474/14/124

Sokunbi, O., Ganiyu, K., Fatimah, G. (2015). Effects of acupuncture, core-stability exercises, and treadmill walking exercises in treating a patient with postsurgical lumbar disc herniation: a clinical case report. J Acupunct Meridian Stud 2015;8(1):48e52

Xiaoping, Q., Qiannan, D., Yanhong, D., Yun, L. (2019). Decoction combined with individualized nursing on lumbar intervertebral disc herniation. Boletín De Malariología Y Salud Ambiental 2019, Vol. 59 (3): 276-280 276 Xiaoli, G., Xiaohua, H., Shan, D., Shumei, C. (2019). Rehabilitation nursing for patient rehabilitation after minimally invasive spine surgery. Int J Clin Exp Med 2019;12(3):2450-2455 Available at: www.ijcem.com / ISSN:1940-5901/IJCEM0085631 